\title{
Efecto del contenido de la humedad en la elaboración de lombriabonos.
}

\author{
J. F. Quant Bermúdez y A. Driutti
}

Instituto Agrotécnico "Pedro M. Fuentes Godo", UNNE, Las Heras 727 , (3500) Resistencia, Chaco, Argentina.

Según la información disponible los tenores óptimos de humedad, sobre la base del peso material, oscilan entre el 50 y el $85 \%$ e incluso hasta próximo a saturación, según clase y acondicionamiento de la materia prima. Conforme a nuestra experiencia de campo nos pareció interesante disponer de información directa y más acotada del efecto de este factor en la preparación del lombriabono. Se partió de un estiércol de corral vacuno con bostas frescas y de un buen grado de pureza proveniente de las vacas en ordeñe de la Escuela de Agricultura (ERAGIA) dependiente de la Facultad de Ciencias Agrarias, UNNE.

Antes de su empleo, una vez secas al aire, se pasa por molino a martillo y por tamiz de malla de $1 \mathrm{~cm}^{2}$. Luego de homogeneizar el material se cargaron y pesaron en forma sucesiva diez porciones de $1000 \mathrm{ml}$, cuyo peso promedio fue de $448 \mathrm{~g} .+0,1$. En base a dicho peso se prepararon 8 unidades en envases de telgopor de $1700 \mathrm{ml}$ de capacidad total; con los cuales, se arma una serie creciente con el agregado de $0,200,300,400,500,600,800$ y $1000 \mathrm{ml}$ de agua corriente. No se hicieron repeticiones. Luego, se registró el peso, aproximación $0,1 \mathrm{~g}$. y se mantuvo a temperatura de laboratorio entre el 07/04 hasta el 29/05/99 (52 dias) con reposición semanal del agua perdida. Durante esta etapa se constató escape por drenaje en 80 $\%$ v/v y en $100 \%$ v/v donde además hubo fuerte producción de gases. El 29/05/99 cada uno de los recipientes se dividieron en dos mediante pesadas y previa homogeneización del contenido de cada uno. Luego de la identificación se armó una serie "sin lombrices" y otra con la incorporación de diez lombrices (Eiseinia foetida) de tamaño mediano $(3-4 \mathrm{~cm})$, sin clitelo evidente, obtenidas de nuestro lombricero mediante diez extracciones sucesivas y agregadas posteriormente al azar, una lombriz en cada extracción. Las dos series continuaron a temperatura de laboratorio $y$, semanalmente, hasta el
07/09/99 (101 dias) se repuso la humedad mediante pesadas. Se efectuaron observaciones cualitativas sobre la evolución del material y de las lombrices. El 06/09/99, a los 100 dias las lombrices originales son lavadas y secadas entre repasadores de algodón y pesadas con aproximación de + 0,1 g. El 29/09/99, a los 123 días, se efectuó la observación final y las 10 lombrices se pesaron con una precisión de $+0,1 \mathrm{mg}$. Luego de extraidas las lombrices y las cápsulas ambas series fueron secadas al aire y homogeneizadas cada tratamiento se dividió en tres porciones para su análisis por triplicado. Las determinaciones fueron: $\mathrm{pH}$ actual 1:2,5, conductividad en pasta saturada, carbono orgánico-Walkley y Black modificado, nitrógeno total por semi-microKjeldhal, N-NO3 fenol-disulfónico, P Bray-Kurtz №1, K fotometría de llama, $\mathrm{Ca}$ y $\mathrm{Mg}$ asimilables por complejometría mediante extracción ácida $(0,05 \mathrm{~N} \mathrm{HCl}+0,025 \mathrm{~N} \mathrm{H} 2 \mathrm{SO} 4)$.

En el cuadro №1 se registraron las principales observaciones efectuadas en el transcurso del ensayo de laboratorio. No se incluye el tratamiento $0 \%$ porque no albergó lombrices. 30/06/99 se registró la aparición del "clitelo " y las lombrices de mayor tamaño correspondieron a los niveles del 60,80 y $100 \%$ en los que, además, se observó deposición del material sobre las paredes del recipiente y en cantidad creciente conforme transcurren los dias $y$, por otra parte, se formaron islotes que no se anegaron con el agua de reposición. El 06/07, a los 38 dias de incorporadas las lombrices se trató de constatar su número. El 15/07 se apreció deposición de "cast" sobre la tapa de los recipientes con tenores del 20 al $50 \%$. El 02/08 a los 65 días se puso atención al "clitelo". El 06/09 y 29/09 a los 100 y 123 dias se efectuaron pesadas de las lombrices originales. Con relación a los datos presentados en el Cuadro №1 se observó que:

Cuadro $\mathrm{N}^{*} 1 \quad$ Observaciones sobre el ensayo de laboratorio

\begin{tabular}{|c|c|c|c|c|c|}
\hline SERIE & $06 / 07$ & & $02 / 08 / 99$ & $06 / 09 / 99$ & $29 / 09 / 99$ \\
\hline $20 \%$ & 10 & & $\begin{array}{l}10 \text { grandes, solo una } \\
\text { ciclitelo incipicnte }\end{array}$ & $\begin{array}{l}\text { 10, no se observa clitelo } X= \\
0.50 \mathrm{~g} \text {. }\end{array}$ & $\begin{array}{l}\text { 10, sin clitelo tierra adheridi } \\
\text { al cuerpo. } \\
x=364 \text { mg }\end{array}$ \\
\hline $30 \% v v$ & $10 \mathrm{y}$ & 33 "cocones" & $\begin{array}{l}10 \text { un poco más } \\
\text { grandes, todas con } \\
\text { clitelo. lay un cocon y } \\
2 \text { I. chicas. } \\
\end{array}$ & $\begin{array}{l}10 \text { grandes ciclisclo, } 57 \text { chicas } \\
\text { y } 5 \text { huevers } X=0.5 \mathrm{~g} \text {. }\end{array}$ & $\begin{array}{l}10 \text { colus oscuro. sin clitelo, } \\
114 \mathrm{de} 1 \mathrm{~cm} \text {, muchas mas } \\
\text { chiquita y } 28 \text { huevos. } X=5.4 \mathrm{x} \\
\text { my }\end{array}$ \\
\hline $40 \% \mathrm{v} / \mathrm{v}$ & 10 & & $\begin{array}{l}10 \text { grandes. con clitclo } \\
\text { más marcado que las } \\
\text { del } 30 \% \text {. }\end{array}$ & $\begin{array}{l}10 \text { grandes ciclitelo. No se } \\
\text { obs. lom. chicas ni corones. } \mathrm{X} \\
=0.63 \mathrm{~g} \text {. }\end{array}$ & $\begin{array}{l}\text { 10, salen limpias cic clicto. } \\
X=576 \mathrm{mg} \text {. }\end{array}$ \\
\hline $50 \% \mathrm{nv}$ & 10 & & $\begin{array}{l}10, \text { todas con clitelo } \\
\text { bien hinchato. }\end{array}$ & $\begin{array}{l}10, \text { grandes con clitele. } \\
X=0, \mathrm{R} \text { g. }\end{array}$ & $\begin{array}{l}\text { 10, salen cmbarradas, } \\
\text { ciclitelo. } X=640 \mathrm{mg} \text {. }\end{array}$ \\
\hline $601 \%$ viv & 10 & & $\begin{array}{l}\text { 10. 1odals con clitelo } \\
\text { bien himchado. Hay } 3 \text {-4 } \\
\text { que aparcecen mas largas }\end{array}$ & 10. todas con ctitelo $x=0,89 \mathrm{~g}$ & $\begin{array}{l}\text { 10, cmbarradas c/clitclo, } \\
x=970 \mathrm{mg}\end{array}$ \\
\hline $80 \% \mathrm{viv}$ & 10 & & $\begin{array}{l}10 . \text { ludas con clitelo } \\
\text { bicn hinchades }\end{array}$ & 10, lodas con clitelo $x=0,92 \mathrm{~g}$ & $\begin{array}{l}\text { 10,con clitelo muy hinchado } \\
\text { y cmbarado. } X=965 \mathrm{mg}\end{array}$ \\
\hline $100 \% \mathrm{v} / \mathrm{v}$ & 10 & & 10. id ant. & 10. wodas con clizelo $x=1.1 \mathrm{~g}$. & $\begin{array}{l}10, \text { wodas c/clitelo } \mathrm{y} \\
\text { conharradas } \mathrm{x}=977 \mathrm{mg}\end{array}$ \\
\hline
\end{tabular}


1) Tanto por falta como exceso de agua no existe reproducción. En esta prueba ello ocurre solamente en el tratamiento iniciado al nivel del $30 \% \mathrm{v} / \mathrm{v}(67 \% \mathrm{p} / \mathrm{p}$ seco y 40,1 p/p húmedo). Por ello, con el mismo material se hizo otra prueba desde el $18 / 10$ al 29/12/99 (72 dias) con el 30 y $45 \% \mathrm{v} / \mathrm{v}$; en esta oportunidad, con el $30 \%$ inicial no hubo reproducción y el peso promedio de las lombrices fue de $377 \mathrm{mg}$ y, en cambio, si con el $45 \%$ inicial y el peso alcanzó los $530 \mathrm{mgr}$. La discrepancia puede explicarse porque en el transcurso del ensayo existe disminución de volumen y, por ende, se modifica la relación porcentual del v/v; por ejemplo, para el mismo $30 \% \mathrm{v} / \mathrm{v}$ en el ensayo original las lombrices fueron extraídas limpias; en cambio, en el segundo salieron con tierra adherida al cuerpo. Por ello, estimamos que una expresión más correcta sería basarse en el peso, aunque sea más práctico trabajar sobre la base de volúmenes.

2) Cuanto mayor es la humedad mayor es la persistencia y desarrollo visual del clitelo, como así también la consistencia del cuerpo de la lombriz que se vuelve muy firme al apretarla entre los dedos.

3) A mayor contenido de humedad mayor peso de las lombrices, estabilizándose, en esta prueba, a partir del $60 \%$ v/v "inicial" Debe recordarse que con el 80 y 100 se produjo pérdida de líquido por drenaje que no pudo ser controlado.

Con respecto a las características químicas de los materiales convienen recordar qué "sin lombrices" sufrió un verdadero proceso de "compostaje" con diferentes tenores de humedad; en cambio, "con lombrices" se elaboró un lombriabono. También, es conveniente aclarar que los diferentes porcentajes de agua $(\mathrm{v} / \mathrm{v})$ tienen significado como valor "inicial" porque con los materiales orgánicos siempre hay reducción de volumen durante el proceso $y$, además, en nuestro ensayo se produjeron pérdidas por drenaje que no fueron ni anuladas ni equilibradas.

De los resultados del Cuadro №2 (Anexo) se observó que:

1) Con lombrices los valores de $\mathrm{pH}$ disminuyeron hasta el $40 \%$; en cambio, aunque con pocas diferencias entre ellos, se incrementa a partir del $50 \%$. Sin lombrices es semejante pero hasta el $30 \%$. Curiosamente, esta serie arroja valores mayores que la otra hasta el $40 \%$ y menores del $50 \%$ en adelante. Esto puede estar vinculado con el potencial redox del medio y a la actividad de las lombrices, tales como sus excreciones.

2) La conductividad eléctrica disminuye conforme aumenta el nivel hidrico, probablemente por efecto "dilución". Cabe remarcar que existió reproducción de las lombrices con 4,53 $\mathrm{mmhos} / \mathrm{cm}$ pero con un $\mathrm{pH}$ de 7,03 unidades. En general la actividad de las lombrices favorecieron la conductividad.

3) Los menores valores de $\mathrm{pH}$ con respecto al testigo podrian atribuirse a la disociación del ácido nitrico producido en el transcurso de la mineralización.

4) Para el carbono orgánico la mineralización máxima ocurre al $50 \%$ con lombrices y al $40 \%$ sin ellas; luego, tanto por falta como por exceso disminuye. Salvo en el nivel $100 \%$, las lombrices favorecieron la oxidación o consumo del carbono orgánico.

5) Para el $N$ total se registra un incremento en los primeros niveles $(20$ y $30 \%$ ) con respecto al testigo que podría atribuirse a un proceso de fijación biológica; en los otros niveles hay disminución y es concomitante con la mineralización de la materia orgánica. Con lombrices los niveles son mayores hasta el $50 \%$; luego, ocurre lo inverso y que podría vincularse a una supuesta mayor aireación por las lombrices.

6) Los tenores de fósforo aparecen un poco más altos que los del testigo hasta el nivel del $60 \%$ inclusive y que podría deberse a la mineralización del material. No aparecen diferencias llamativas entre ambas series con y sin lombrices.

7) Con relación al potasio asimilable en la serie con lombrices en los tres primeros niveles (20, 30 y $40 \%$ ) superan en algo más del $10 \%$ al testigo; en cambio, en los restantes están por debajo en alrededor de un $15 \%$. No se observan mayores diferencias entre las dos series. Estos resultados podrian asociarse a la mineralización del estiércol y los niveles hidricos.

8) En general con la incubación se solubilizó calcio, especialmente en los tenores más bajos, 20 y $30 \%$. No se aprecia un efecto evidente por las lombrices.

9) Con relación al $\mathrm{Mg}$ asimilable no se registran diferencias consistentes con respecto al testigo sin incubar.

10) El contenido de $\mathrm{N}-\mathrm{NO} 3$ al final del ensayo constituye el parámetro más revelador y contrastante: con lombrices hay abundantes nitratos hasta en el $40 \%$ y el valor máximo ocurre con el $20 \%$; sin lombrices, el tope se registra al $30 \%$, luego cae abruptamente.

11) El análisis de la regresión polinomial para los parámetros del cuadro №2 con los diferentes niveles hídricos solamente registró una regresión lineal significativa en los casos de $\mathrm{C}$ orgánico con lombrices y para K asimilable sin lombrices.

Con este ensayo sin repeticiones podemos concluir que:

1) El nivel de humedad es un factor importante en el crecimiento de las lombrices expresado en su peso vivo y decisivo en la reproducción dentro de límites estrechos. Para las condiciones del ensayo y del sustrato dicho nivel fue del $30 \% \mathrm{v} / \mathrm{v}$ inicial. Para mayores precisiones se deberia trabajar en base al peso.

2) La influencia de las lombrices sobre el sustrato se hace evidente en condiciones aeróbicas. En este ensayo entre el 20 y $40 \%$ v/v ïnicial" ocurririan los procesos bioquímicos más adecuados par la obtención de mejores lombriabonos. 
3) El lombriabono más adecuado fue el obtenido con el $30 \%$ de humedad y se evidencia en su $\mathrm{pH}$, el tenor de carbono orgánico, nitrógeno total, relación $\mathrm{C} / \mathrm{N}$ y grado de mineralización que se traduce en la cantidad de nutrientes asimilables.

Cuadro $\mathrm{N}^{\circ} 2$

lombrices (s/l)

\begin{tabular}{|c|c|c|c|c|c|c|c|c|c|c|c|c|c|c|c|c|c|c|c|c|}
\hline \multirow[t]{3}{*}{$\begin{array}{l}\% \\
\text { agua } \\
v / v\end{array}$} & \multirow{2}{*}{\multicolumn{2}{|c|}{ PH }} & \multirow{2}{*}{\multicolumn{2}{|c|}{$\begin{array}{l}\Omega \\
\mathrm{mmhos} / \mathrm{cm}\end{array}$}} & \multirow{2}{*}{\multicolumn{2}{|c|}{$\begin{array}{l}\text { C } \\
\text { org } \\
\text { g.\% }\end{array}$}} & \multirow{2}{*}{\multicolumn{2}{|c|}{$\begin{array}{l}\mathrm{Nt} \\
\mathrm{mg} \%\end{array}$}} & \multirow{2}{*}{\multicolumn{2}{|c|}{$C N$}} & \multicolumn{6}{|c|}{ Ppm } & \multicolumn{3}{|c|}{ meq $/ 100 \mathrm{~g}$} & \\
\hline & & & & & & & & & & & \multicolumn{2}{|c|}{$\mathrm{N} N \mathrm{NO}^{3}$} & \multicolumn{2}{|c|}{$\mathrm{P}(\mathrm{ByK})$} & \multicolumn{2}{|l|}{$\mathrm{K}$} & \multicolumn{2}{|l|}{$\mathrm{Ca}$} & \multicolumn{2}{|l|}{$\mathrm{Mg}$} \\
\hline & $\mathrm{C} / 1$ & $\mathrm{~S} \mathbf{1}$ & $\mathrm{C} / \mathrm{l}$ & $5 /$ & $\mathrm{C} / \mathrm{I}$ & $\mathbf{S} / 1$ & $\mathrm{C} / \mathrm{I}$ & SI & $\mathrm{C} / \mathrm{I}$ & $\mathrm{S} / \mathrm{H}$ & $\mathrm{C} / \mathrm{I}$ & $\mathbf{S} / 1$ & $\mathrm{C} / 1$ & $\mathrm{~S} /$ & $\mathrm{C} / \mathrm{l}$ & Sil & $\mathrm{C} / \mathrm{I}$ & $\mathbf{S} / 1$ & C.I & Sil \\
\hline $\begin{array}{l}0 \\
\text { testigo }\end{array}$ & \multicolumn{2}{|r|}{8.22} & \multicolumn{2}{|r|}{2,13} & & 10.8 & \multicolumn{2}{|c|}{431} & \multicolumn{2}{|c|}{25} & \multicolumn{2}{|c|}{19} & \multicolumn{2}{|c|}{1890} & \multicolumn{2}{|c|}{6459} & \multicolumn{2}{|c|}{46.2} & \multicolumn{2}{|r|}{32.2} \\
\hline 20 & 7.17 & 7.53 & 4.43 & 3.50 & 8.67 & 9.65 & 538 & 485 & 16.0 & 20.1 & 1505 & 617 & 2065 & 2258 & 7390 & 7445 & 56.8 & 54.9 & 32.4 & 31.6 \\
\hline 30 & 7.03 & 7.31 & 4.53 & 3.65 & 9.06 & 10.23 & 538 & 431 & 16.8 & 23.9 & 1297 & 903 & 2310 & 2398 & 7226 & 7226 & $\$ 6.6$ & 56.2 & \begin{tabular}{|l|l}
34.3 \\
\end{tabular} & 33.2 \\
\hline 40 & 7.60 & 8.73 & 3.50 & 2.00 & 7.83 & 8.70 & 395 & 377 & 19.8 & 23.1 & 718 & 11 & 2030 & 2100 & 7323 & 6076 & 48.3 & 41.7 & 29.9 & 26.3 \\
\hline 50 & 8.68 & 8.71 & 1.83 & 1.77 & 7,40 & 9.40 & 377 & 343 & 19.6 & \begin{tabular}{|l|}
27.7 \\
\end{tabular} & 16 & 13 & 2100 & 2083 & 5449 & 5494 & 42.7 & 47.4 & 27.4 & 28.8 \\
\hline 60 & 8.85 & 8.57 & 1.47 & 1.23 & 9.00 & 9.8 & 377 & 413 & 239 & 23.7 & 10 & 12 & 2170 & 2065 & 5693 & 5419 & 48.1 & 49.2 & 30.7 & 31.5 \\
\hline 80 & 8.83 & $8.7 \overline{4}$ & 1.33 & 1.40 & 9.80 & 9.85 & 377 & 431 & 25.9 & 23 & 10 & 11 & 1870 & 1855 & 5638 & 5638 & 510 & 42.7 & 34.7 & 25.4 \\
\hline 100 & 8.76 & 8.68 & 1.27 & 1.30 & 9.90 & 9.77 & 395 & 431 & 25.0 & 24.7 & 9 & 13 & 1908 & 1820 & 5310 & $\$ 419$ & 45.9 & 43.7 & 29.7 & 28.8 \\
\hline
\end{tabular}

